\title{
International Development, Emerging Diseases, and Ecohealth
}

For nearly half a century, the medical community in most developed countries has focused on emerging infectious diseases (EIDs) as a key threat to global health. EIDs are thought to primarily originate in tropical regions where wildlife biodiversity and human population density are high (Jones et al. 2008). Simultaneously, most of the countries that are home to the bulk of the world's biodiversity are also among its poorest economies. These regions are under-resourced in public health capacities, therefore limiting local ability to cope with day-to-day endemic disease threats as well as delaying the identification and response to emerging pathogens. Broadly speaking, poor public health infrastructure remains a key impediment to global development and may contribute to a disease-driven 'poverty trap' (Bonds et al. 2010) in which poor health actually impedes economic growth and poverty reduction. How do we reconcile the developed world's concern about emerging diseases, and a critical need for international development resources to tackle all infectious disease threats, emerging and endemic?

Global resources allocated to development assistance for health have risen substantially over the past decade (Ravishankar et al. 2009), though the impact of donor aid on government health spending in countries receiving aid has been questioned in recent literature (Lu et al. 2010). The vast majority of health spending in developing countries comes from aid budgets, and the vast majority of these budgets are earmarked for AIDS, malaria, TB, and polio. Spending on water, sanitation, and basic public health infrastructure is significantly less than on other health issues. Much attention has been focused on these vertical (single disease focus) versus horizontal (broad public health focus) approaches to spending, stemming from continued debates as to whether funds for specific issues such as HIV/AIDS displaces funds from other public health programs (Shiffman 2007). Yet while awareness of the need to combine different facets of health into an integrated approach has long been gaining traction among researchers, it has yet to be fully absorbed into policy and government-level decision-making.

An integrated, "ecohealth" approach is of particular import to developing countries. The highest rates of on-going destructive land-use change and biodiversity loss are occurring in the world's lesser economically developed countries, though emerging economies, such as China, Brazil and India are also at the forefront of these changes. Many of these countries contain public health systems supported fully or partially by foreign development aid, which is often used to strengthen nascent health systems. At the same time, separate initiatives, from separate funding streams (e.g. World Organization for Animal Health [OIE] and FAO), seek to develop agricultural practices, monitor livestock health and preserve biodiversity. As such, we suggest that foreign aid could be streamlined and made more effective by integrating development objectives, particularly when they relate to human, animal, and wildlife health-in other words, by adopting an ecohealth approach at an international development policy level.

As part of this over-arching strategy, specific initiatives within the development toolkit could be broadened to deal with the challenges of creating sustainable and integrated health systems. One such example is pandemic prevention. Outbreaks of infectious diseases generally have the most observable economic impact on developed countries, yet commonly emerge in developing regions. Recent research (Bogich et al. 2012) has pointed to breakdowns in national 
public health infrastructure as the dominant source of outbreak events, far overshadowing more widely covered causes such as the bushmeat trade. Despite such mundane origins, the effects of outbreaks are often felt as emergency events, and, correspondingly, efforts to control outbreaks are funded as such. In this way, development aid for pandemic prevention is still channeled primarily through emergency relief funds, rather than being used to strengthen local monitoring networks and public health systems to deal with emerging diseases at the point of origin. Given that most recent pandemics have zoonotic beginnings, the relevance of an ecohealth approach to pandemic prevention is clear and critical.

An ecohealth approach to development also makes intuitive sense economically. Separate funding streams currently provide money for development initiatives relating to human public health versus animal/veterinary diseases. Further, much attention has been focused on high-tech advances (rather than basic public health infrastructure) with the recent proliferation of BSL-3 and BSL-4 laboratories as an example (Dixon 2005). However, in the developing world high-cost laboratory systems are often not economically sustainable, and low-cost, lower-tech security upgrades may actually have a greater long-term impact on laboratory safety and pathogen security. As such, the emphasis should be on pragmatic initiatives which cater to the needs and capabilities of each country or region, rather than insisting on "one size fits all" solutions.

To parallel an on-going trend within human infectious disease control projects, the aim should be to create synergy through integration: the sum of the impact of two projects may be greater when combined. In the human disease control analogy, efforts have been made to maximize the efficiency of separate control strategies by bringing them under the same umbrella initiative. USAID has set the precedent for such programs, first funding a global avian influenza network and now an emerging pandemic threats program, with the aim of integrating animal and human health, facilitating technological and laboratory advances while building local capacity (Mazet et al. 2011). On a national scale, USAID has supported Uganda in recently combining all of its neglected tropical disease programs into one coordinated initiative (Linehan et al. 2011). Likewise, with support from various US agencies (including the Department of State, the Department of Defense and the Centers for Disease Control and Prevention), Kenya launched a Zoonotic Disease Unit in October 2012, whereby epidemiologists from both the Ministry of
Agriculture and Livestock and the Ministry of Public Health and Sanitation sit together to monitor and respond to disease outbreaks (Lore 2012). As such, now would be an opportune time for future health development aid to both Uganda and Kenya to encourage joint monitoring of veterinary and wildlife health for a more fully integrated approach to control of these, often zoonotic, diseases.

To conclude: wherefore ecohealth and development? It has been more than 50 years since Dr Calvin Schwabe first coined the term "One Medicine" (also known as "One Health"), thus conceptually bringing together the joint aims of wildlife and human health; in the same period of time, US $\$ 2.3$ trillion has been spent by the USA alone on foreign aid. 'Operationalizing' One Health seems improbable without umbrella government support and a specific defined budget among the agencies, as opposed to the current situation of each relevant agency (e.g. Ministries of Health, Agriculture, and the Environment) competing for funds. An ecohealth systems approach is needed to reunite development with health, both animal and human. Furthermore, ecohealth advocates should strategize to collaborate with economists, to provide a clear model of the costs and benefits associated with an integrated development approach. Numbers talk, and such an analysis would provide further ammunition for bringing ecohealth as a development strategy to the highest policy-making tables.

Claire J. Standley, \& Tiffany L. Bogich,

Department of Ecology \& Evolutionary Biology

Princeton University, Princeton, NJ, USA

e-mail: tbogich@princeton.edu.

\section{REFERENCES}

Bogich TL, Chunara R, Scales D, Chan E, Pinheiro LC, Chmura AA, Carroll D, Daszak P, Brownstein JS (2012) Preventing pandemics via international development: a systems approach. PLoS Med 9:e1001354

Bonds MH, Keenan DC, Rohani P, Sachs JD (2010) Poverty trap formed by the ecology of infectious diseases. Proceedings of the Royal Society B-Biological Sciences 277:1185-1192

Dixon B (2005) Worries over bioterrorism push. Current Biology 15:R229-R230

Jones KE, Patel N, Levy M, Storeygard A, Balk D, Gittleman JL, Daszak P (2008) Global trends in emerging infectious diseases. Nature 451:990-994

Linehan M, Hanson C, Weaver A, Baker M, Kabore A, Zoerhoff KL, Sankara D, Torres S, Ottesen EA (2011) Integrated implementation of programs targeting neglected tropical diseases through preventive chemotherapy: proving the feasibility at 
national scale. American Journal of Tropical Medicine and Hygiene 84:5-14

Lore T (2012) Kenya launches zoonotic disease unit to improve prevention and control of zoonoses. AgHealth: Prevention and control of agriculture-associated diseases http://aghealth.word press.com/2012/10/08/kenya-launches-zoonotic-disease-unitto-improve-prevention-and-control-of-zoonoses/.

Lu C, Schneider MT, Gubbins P, Leach-Kemon K, Jamison D, Murray CJL (2010) Public financing of health in developing countries: a cross-national systematic analysis. The Lancet 375:1375-1387

Mazet J, Daszak P, Goldstein T, Johnson C, Fair J, Wolfe N, Joly D, Smith K, Morse S, Karesh W (2011) Predicting pandemics:
Using a one health approach to identify and mitigate the emergence and spread of zoonoses from wildlife. Ecohealth 7:S137-S137

Ravishankar N, Gubbins P, Cooley RJ (2009) Financing of global health: tracking development assistance for health from 1990 to 2007. Lancet 373:2113-2124

Shiffman J (2007) Has donor prioritization of HIV/AIDS displaced aid for other health issues? Health Policy and Planning 23:95-100

Published online: February 14, 2013 